\title{
DESIGN ISSUES AND CHALLENGES OF RELIABLE AND SECURE TRANSMISSION OF MEDICAL IMAGES
}

\author{
Mohd.Javed Khan ${ }^{1}$, Asok Bhattacharyya ${ }^{2}$, Monuwer Alam ${ }^{3}$ \\ ${ }^{I}$ Dept. Electronics \& Comm. Engg, Integral University, Lucknow, India \\ ${ }^{2}$ Dept. Electronics \& Comm. Engg, Delhi Technical University, Delhi, India \\ ${ }^{3}$ Dept. Electronics \& Comm. Engg, Integral University, Lucknow, India
}

\begin{abstract}
The transmission of digital image data now a day's become a routine and necessity of our society. The amount of digital medical data is increasing rapidly on internet. Now it is become necessity of the secure and reliable digital image data transfer for the fast and efficient diagnosis in the medical world. A lot of data is to be transferred between hospital where the major portion of the data is the medical images, patient history, and pervious diagnosis record. Digital Imaging and communication in medicine (DICOM) standard are used to solve this problem where the patient records are attached. To make the transmission robust the patient record are embedded in the medical image using digital water marking technique for the reliable and secure transmission. The various type of noise may corrupt the medical image data during transmission and even storage media may also be affected. Using Error Control Coding Techniques to Encode the medical images may one of the possible solutions for this problem.
\end{abstract}

Keywords—DICOM, ECC, Water Marking, Discrete Wavlet Transform

$* * *$

\section{INTRODUCTION}

For the fast and efficient diagnosis a huge amount of data is required to be transmitted over wireless or internet in medical world. The major portions of the data which we have to share between hospitals are the medical images and diagnosis records (i.e. patient history). If the patient basic details and medical images are stored in a separate data base with different file names may lead to mishandling such as unauthorized access. DICOM standard are used to solve the above problem .In DICOM images the header are filled with ASCII character of patient record are normally used in most hospital all over the world. But in this system since there are no security feature the unauthorized access and the loss of header information will create the problem. For the data transmission and storage the digital water marking technique may improve the DICOM standard. In watermarking technique the patient record are embedded in the medical images which will reduce the possibility of unauthorized access and it does not need any separate header information for the patient record which also improves if the header information is lost during transmission.

Digital watermarking is a technique in which hidden information (also known as watermark) is inserted in to the original data (multimedia data or cover media).Water mark may be in image ,text , audio or video form .By inserting or embedding the watermark with specific algorithm original data is slightly modified[1-3]. The modified data is known as watermarked media. So there will be little or no perceptible difference between original media and watermarked media content [4].

Watermark embedding techniques are divided into two basic categories-

1.By Embedding the watermark into the cover signal through the LSB (least significant bit ).In it LSB of the cover signal is replaced by the watermark to get the watermarked image .It has less complexity and can be easily implemented but it is not robust to any types of attacks.

2.By Embedding the watermark in to the transform domain of cover image .Transform domain or frequency domain is obtained by taking the DFT(discrete Fourier Transform) or DCT (Discrete Cosine Transform ) or DWT (Discrete Wavelet Transform ).After this low frequency component are modified to obtained the watermarked image.

\section{RELATED WORK}

Watermarking technique is used in compact storage and transmission of patient text information or bio -signals and medical images.

In Spatial domain watermarking technique ,patient information is embedded into the medical images[5-6] and Percentage normalized root mean square error (\%NRMSE) is computed for original and watermarked images.ECC techniques is used to recover the watermarked image even though it get corrupted during storage and transmission[7-8]. 
In Transform domain watermarking technique ,patient information is embedded into the medical images[8]. Percentage normalized root mean square error (\%NRMSE) will be compared in DFT, DCT and DWT domain for medical images. There is a slight loss of information in the original image.

The \%NRMSE between original image and watermarked image will be computed and analyzed.

Reversible watermarking technique is used to embedded patient text information into the medical images. The $\%$ NRMSE between original image and watermarked image will be computed for medical images. Then ECC technique is used to encode the watermarked image and recover it even though is corrupted during storage and transmission.

\section{PROPOSED METHODOLOY}

Figure 1 shows the proposed scheme in which patient text information is embedded in medical images and then it is encoded with ECC the technique before transmission.

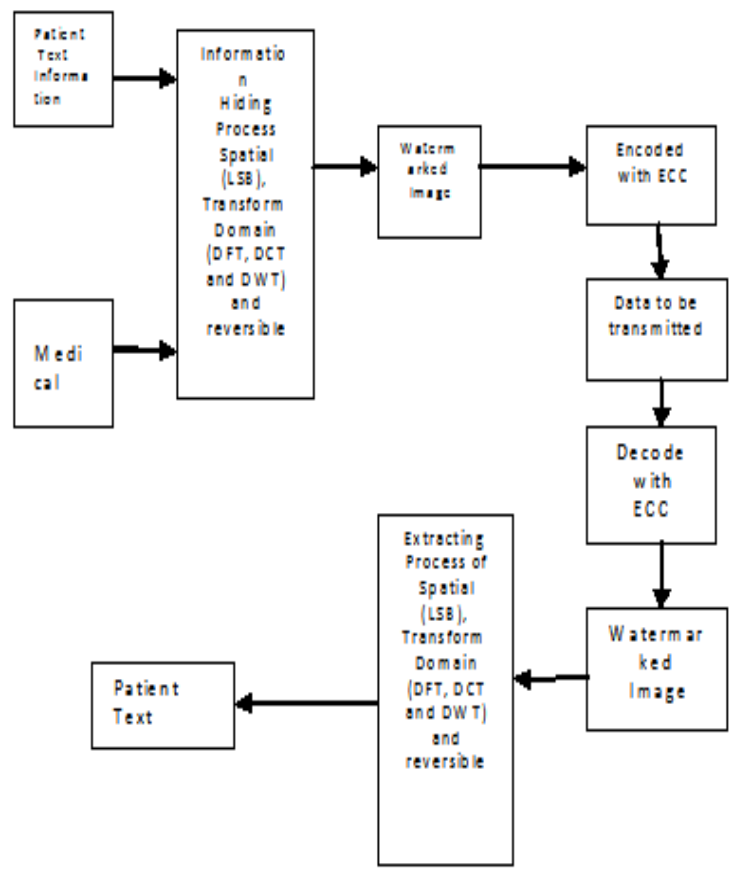

Fig-1 Proposed Scheme for the integrated transmission and storage of text information with medical image and extracted patient text information from the watermarked image

Embedding is done by using spatial (LSB) or by Transform domain (DFT, DCT and DWT) or by reversible watermarking. And to get the patient text information ,transmitted information is first decoded using ECC and then different type of extracting methods are used .Discrete wavelet Transform will be used to solve out the problem of image compression and decompression and Arnold Scrambling algorithm will be used to solve the watermarking problem. And for Error correction Codes Turbo code is used.

Wavelet compression involves a way of analyzing an uncompressed image in recursive fashion resulting in a series of higher resolution images, each "adding to" the information content in lower resolution image.

There are five steps in compression and decompression technique, those are shown in figure 2 and figure 3 .

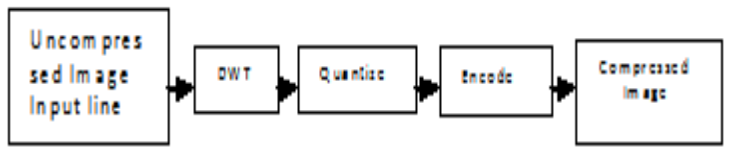

Fig2 Wavelet based image Compression

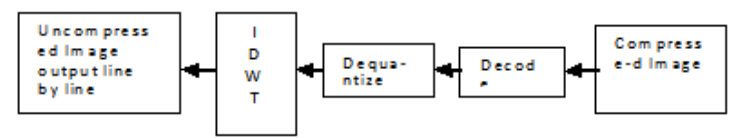

Fig-3 Wavelet based image decompression

Lossless or reversible compression refer to compression technique in which reconstructed data exactly matches the original. Lossless compression denotes compression methods, which give the quantitive bounds on the nature of the loss that is introduced. Such compression techniques provide the guarantee that no pixel difference between the original and compressed image is above a given value.

There are various Loss-Less Compression methods which area. Run-Length encoding

b. Entropy coding

c. Adaptive dictionary algorithm such as LZW (Lempel- Ziv Welch )

d. Deflaction

In this paper Arnold transform is chosen as pretreatment method for watermark signal as it is simple and periodic.

Arnold transformation was proposed by Mathematician Arnold [9] in the study of ergodic theory. If the original image size is $\mathrm{N} \times \mathrm{N}$, and $(\mathrm{x}, \mathrm{y})$ is the pixel coordinate, then after the geometric transformation the pixel is moved to $\left(x^{\prime}, y^{\prime}\right)$. This geometric transformation can be described as follows [10]:

$$
[x y]=[x+y x+2 y] \bmod t
$$

Where " $\mathrm{t}$ " is the width (height) of the watermark, Every pixel in the image is transformed in this formula. After traversing all of the pixels in image, a scrambling image is gained. Transforming an image repeatedly can generate different 
result until reaching the requirement. Due to the Arnold transform periodicity, original image can be recovered.

\section{CONCLUSIONS}

Since in our proposed method the patient text information is interleaved in to the medical images by using Watermarking Technique. This technique will overcome the problem of header loss (Patient basic details) during transmission in conventional DICOM. The watermarking technique also improves the unauthorized access of the data. So the proposed methodology will be suitable for the secure and reliable transmission of medical images, patient history and patient ID over the internet for the fast and efficient diagnosis of patient

\section{REFERENCES}

[1]. Delaigl E J F, Vleeschouwer C D, Macq B.: Watermarking Algorithm Based On A Human Visual Model. J. Signal Processing. 66(5), 319--336(1998).

[2]. SWANSON M D, ZU B, TEWF IKA H.: Robust Data Hiding For Images. J. Proc IEEE 7th Digital Signal Processing Workshop (DSP 96). 9, 37--40(1996).

[3]. Gu-song Hao, Yang Cai, Zhang Haiyu, Liu Xiaoxia.: One Iimproved Arnold Transformation. J. Henan University (Natural Science). 2, (2009).

[4]. Qi Dongxu, Zou Jianchun, Han Xiaoyou.: A New Class of Scrambling Transformation And Its Application In The Image Information Covering. J. Science In China Scrics., 43 (3), 304-312 (2000).

[5]. Baraff D, Witk A, Kass M.: Untangling Cloth. J. ACM Transactions On Graphics. 22(3), 862--870(2003).

[6]. Wang Lulu, Zhang Chong.: Arnold Scrambling Based On Digital Image EncryptionTechnique. J. National Defense Technology Base. 10, (2010).

[7]. Huang Fangyuan.: Arnold Scrambling Based On Image Scrambling Algorithm And Implementation. J. Gui Zhou University (Natural Science). 25(3), (2008).

[8]. Wu Lingling, Zhang Jianwei, Ge qi.: Arnold Transformation And Its Inverse Transformation. J. Micro Computer. 14, (2010).

[9]. Huang Liangyong, Xiao Degui.: The Best Scrambling Degree of Arnold Transformation On Binary Images. J. Computer Applications. 2 (2009)

[10]. G. Coatrieux, H. Main, B. Sankur, Y. Rolland, and R. Collorec. Relevance of watermarking in medical imaging. In IEEE-embs Information Technology Applications in Biomedicine,pages 250-255, Arlington, USA, Nov. 2000. 